\title{
Neutrino Physics: the Roadmap for Precision Physics
}

\author{
O. L. G. Peres \\ Universidade Estadual de Campinas - UNICAMP, Instituto de Física Gleb Wataghin, \\ Departamento de Raios Cósmicos, Caixa Postal 6165, 13083-970 Campinas, SP, Brazil
}

Received on 5 April, 2006

\begin{abstract}
In the last years, we experienced a complete change of the view of weak interaction physics. Robust results from many experiments as Super-Kamiokande, KamLAND, SNO, K2K, show us that the neutrinos have the remarkable phenomena of oscillations, a quantum interference mechanism that operates to distances as large as $100 \mathrm{~km}$ and even bigger distances. From this we know that neutrinos change identity from one flavor to another, as was demonstrated by the joints results of SNO and Super-Kamiokande experiments. We show here the review of latest results of neutrino physics, as for example, the first evidence of neutrinos produced in the core of the earth and the updated results of KamLAND and others. Our understating of all experimental results will completed by the state-of-art of the theoretical effort to understand such phenomena. For the near future, we expect the new generation of precision physics, like the running experiments of MINOS and Double CHOOZ, and the proposals of SADO and ANGRA shed light on unresolved issues such as the CP-violation for neutrinos and the relative magnitude of solar and atmospheric scales.
\end{abstract}

Keywords: Neutrinos; Neutrino oscillation

\section{INTRODUCTION}

In the last years it have been show that a new phenomena established a existence of source of lepton number violation not predicted: neutrino oscillation. We are going to review some of these new information and what we can shed new information about the generation of masses in the standard model. We know from the Standard model of particle physics, that we need three neutrinos: the electron, muon and tau neutrinos [1] which for simplicity and lack of any hint that neutrinos are massless and unmixed. The situation change when Pontecorvo [2] and independently Maki, Nakagawa and Sakata [3] propose that neutrino can have a inequality between the mass basis and the flavor basis, if so then the neutrino can suffer from neutrino oscillation: a given flavor neutrino, let's say electron neutrino as produced in the sun, can converted in way to earth to another neutrinos, muon and tau like neutrinos.

After 50 years of the first detection of neutrinos using a reactor by Cowan and Reines [4] we know know that neutrinos can unraveling many aspects of the nature and one of them is the so called neutrino oscillation.

Many experiments with atmospheric neutrinos [5-9] and with solar neutrinos [10-16] indicate that flavor conversion should occur in nature. We show in Fig. 1 the results that show the disappearance of muon neutrinos for atmospheric neutrinos.

Beside that, the experiment KamLand [17], measured antineutrinos produced by reactors and also saw strong evidences for electron neutrino disappearance. This implies that the picture of neutrino conversions as indicated by solar experi- ments is confirmed beyond doubt. Also the SNO experiment, that measure the charged and neutral interactions of deuterium with electron and all flavors of neutrinos [16] show that the electron disappearance, detected by other experiments, is also accompanied by muon and tauon neutrino conversion. We show in Fig. 2 that the SNO data indicate a electron to muon/tauon neutrino conversion.

Also a accelerator experiment K2K [18] show evidences for muon neutrino disappearance. This confirm the muon neutrino disappearance seen in the atmospheric neutrino SuperKamiokande experiment [8]. Recently the MINOS experiment report also evidence for muon disappearance [19] compatible which was found by Super-Kamiokande experiment.

How do we understand this series of experimental evidences for neutrino disappearance in all these experiments? We will show that a consistent picture emerges when neutrinos have non-vanishing masses and they mix between each other.

\section{NEUTRINO MASS SCHEMES}

When the flavor eigenstates of neutrino are not equal to masses eigenstates as suggested by Pontecorvo and others [2] then is possible to have neutrino conversion as long the mixing angles are not zero and the masses are not degenerated. The most general neutrino evolution include the matter potential (due collective contribution from the medium, so called matter effect) [21]. In this case we can write down the neutrino evolution as

$$
i \frac{d}{d t}\left(\begin{array}{c}
\mathrm{v}_{e} \\
\mathrm{v}_{\mu} \\
\mathrm{v}_{\tau}
\end{array}\right)=\left[U\left(\begin{array}{lll}
0 & 0 & 0 \\
0 & \delta & 0 \\
0 & 0 & \Delta
\end{array}\right) U^{\dagger}+\left(\begin{array}{ccc}
V_{e} & 0 & 0 \\
0 & 0 & 0 \\
0 & 0 & 0
\end{array}\right)\right]\left(\begin{array}{c}
v_{e} \\
v_{\mu} \\
v_{\tau}
\end{array}\right)
$$



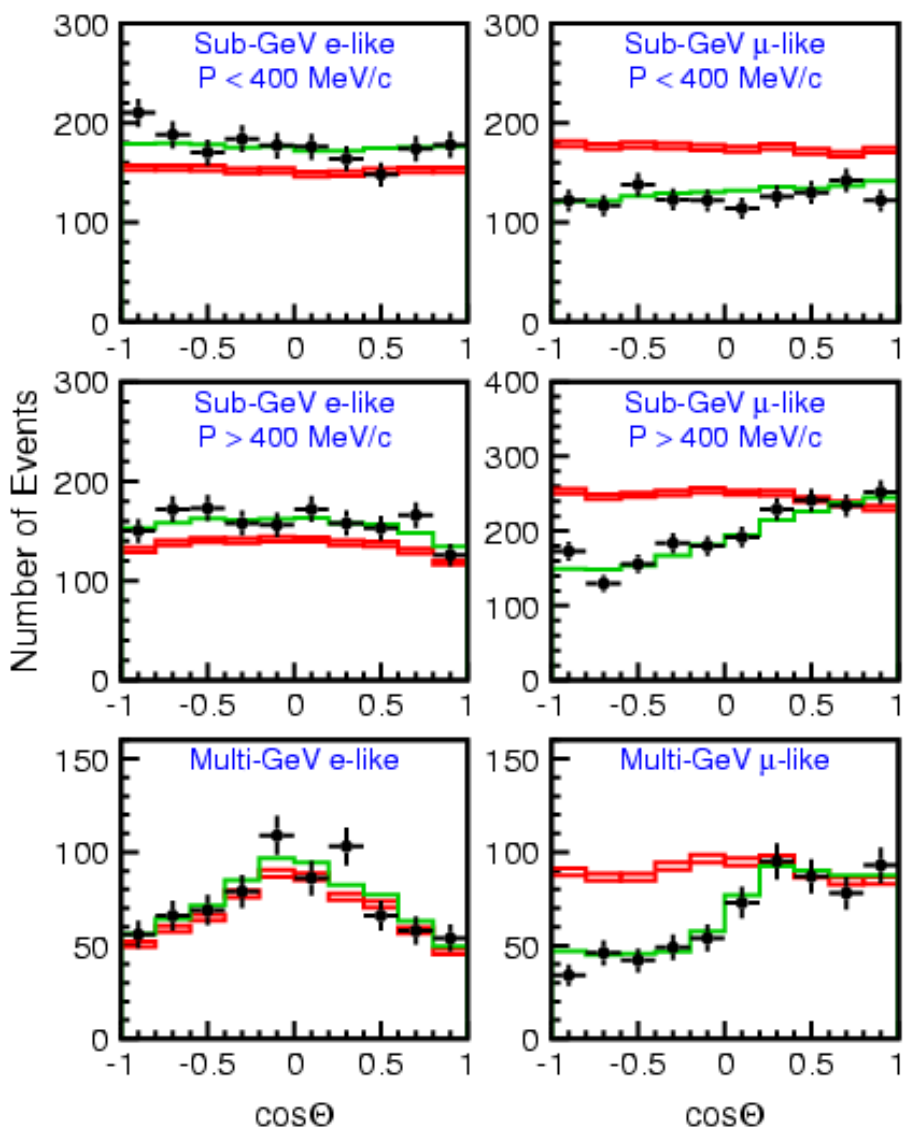

FIG. 1: Atmospheric neutrino data from Super-Kamiokande experiment, the left panel is the electron type events and the the right panel is the muon type events. The energy range increase from top to down. From Ref. [8].

where $\delta=\frac{\Delta \mathrm{m}_{21}^{2}}{2 E}, \Delta=\frac{\Delta \mathrm{m}_{31}^{2}}{2 E}, \Delta m_{i j}^{2} \equiv m_{i}^{2}-m_{j}^{2}$ is the mass squared difference between neutrino families $i$ and $j$, and $\mathrm{U}$ is the mixing matrix as presented in the Particle Data Group (PDG) [20] as given by

$$
U \equiv\left(\begin{array}{ccc}
1 & 0 & 0 \\
0 & c_{23} & s_{23} \\
0 & -s_{23} & c_{23}
\end{array}\right)\left(\begin{array}{ccc}
c_{13} & 0 & s_{13} e^{-i \gamma} \\
0 & 1 & 0 \\
-s_{13} e^{i \gamma} & 0 & c_{13}
\end{array}\right)\left(\begin{array}{ccc}
c_{12} & s_{12} & 0 \\
-s_{12} & c_{12} & 0 \\
0 & 0 & 1
\end{array}\right)
$$

where $c_{i j} \equiv \cos \left(\theta_{i j}\right), s_{i j} \equiv \sin \left(\theta_{i j}\right)$ correspond to cosine, sine of the mixing angle $\theta_{i j}$ between the families $i$ and $j$. The neutrino matter potential is denoted by $V_{e}=G_{F} \sqrt{2} N_{e}(r)$ where $N_{e}(r)$ is the number densities of the electrons. Using this neutrino evolution we are able to find that two scales are relevant for neutrino oscillation, the scale between the first and second family, $\Delta \mathrm{m}_{21}^{2}$ and the scale between the first and second family, $\Delta \mathrm{m}_{31}^{2}$. Also $\gamma$ is the CP violation angle, that for now we will assume to be zero.

The analysis of solar [10-16] as well reactor experiments [17] agree that two states, $l$ and 2 mix strongly and are related with a mass scale $\Delta m_{21}^{2}$. We call this scale, the solar scale, $\Delta m_{\odot}^{2} \equiv \Delta \mathrm{m}_{21}^{2}$ and the mixing angle is called the solar angle, $\tan \left(\theta_{21}\right) \equiv \tan \left(\theta_{\odot}\right)$. From the solar neutrinos experiments we need to have $\Delta m_{21}^{2}>0$. The $68 \%$ C.L. range is

$$
\begin{gathered}
\Delta \mathrm{m}_{\odot}^{2}=8.0_{-0.3}^{+0.4} \times 10^{-5} \mathrm{eV}^{2} \\
\sin ^{2}\left(\theta_{\odot}\right)=0.310 \pm 0.026
\end{gathered}
$$

The allowed region is showed in Fig. 3.

The analysis of atmospheric [5-9] as well accelerator experiments, as K2K [18] and MINOS experiment [19] agree that two states, 2 and 3 mix strongly and are related with a mass scale $\Delta m_{32}^{2}$, that is directly related with scale relevant for atmospheric neutrino experiments. We call this scale, the atmospheric scale, $\Delta m_{\mathrm{atm}}^{2} \equiv \Delta \mathrm{m}_{32}^{2}$ and the mixing angle is called 


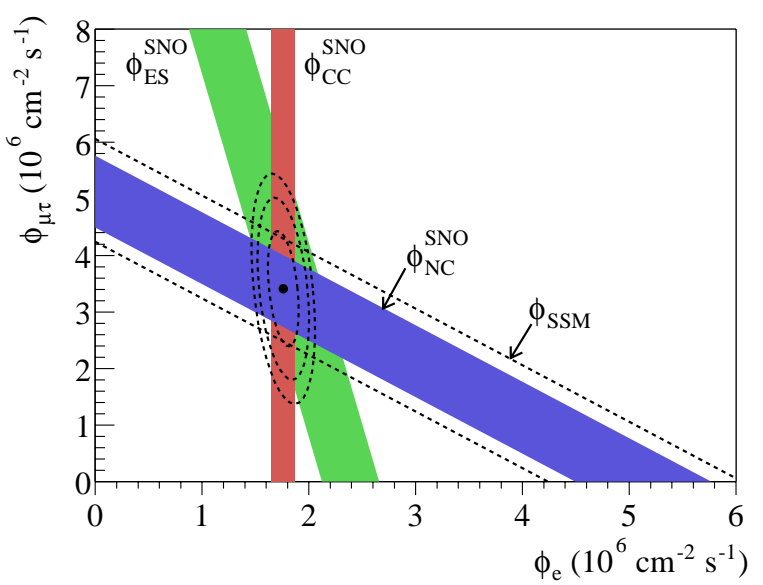

FIG. 2: Flux of ${ }^{8} \mathrm{~B}$ solar neutrinos which are $\mu$ or $\tau$ neutrinos vs flux of electron neutrinos deduced from the neutrino reactions in SNO. The bands denoted by $\Phi_{C C}^{S N O}, \Phi_{E S}^{S N O}$ and $\Phi_{N C}^{S N O}$ are respectively the deuterium charged current, the elastic scattering of electrons and the deuterium neutral current constraints from SNO experiment. From Ref. [16].
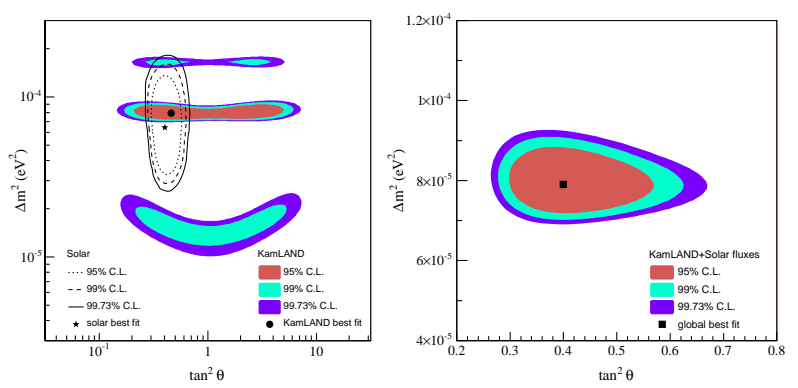

FIG. 3: Allowed regions for $\Delta m_{\odot}^{2}$ and $\tan ^{2}\left(\theta_{\odot}\right)$ from solar experiments and solar+KamLand experiments respectively in the left and right panels. From Ref. [17].

the atmospheric angle, $\sin ^{2}\left(2 \theta_{\text {atm }}\right) \equiv \sin ^{2}\left(2 \theta_{32}\right)$. From the atmospheric and accelerator neutrinos experiments we cannot discriminate between the case $\Delta \mathrm{m}_{32}^{2}>0$ and the case $\Delta \mathrm{m}_{32}^{2}<0$. The values for the atmospheric scale and the atmospheric mixing angle are

$$
\begin{array}{r}
\left|\Delta \mathrm{m}_{\mathrm{atm}}^{2}\right|=3.0_{-1.1}^{+0.3} \times 10^{-3} \mathrm{eV}^{2} \\
\sin ^{2}\left(2 \theta_{\mathrm{atm}}\right)>0.9
\end{array}
$$

The allowed region is showed in Fig. 4. Due the blindness of the signal of $\Delta \mathrm{m}_{\mathrm{atm}}^{2}$ value then you can have two mass hierarchies, as seen in Fig. 5.

Other very important information is the value of angle $\theta_{13}$, which we have only a upper limit. The reactor experiment CHOOZ [22] put a strong constrain in the $\Delta m_{31}^{2}-\theta_{13}$ plane as shown in Fig. 6. We get that

$$
\sin ^{2}\left(2 \theta_{31}\right)<0.12 \text { for } \Delta \mathrm{m}_{\mathrm{atm}}^{2}=3.0 \times 10^{-3} \mathrm{eV}^{2} \text {. }
$$

Future experiments can be able to pinpoint the value of $\theta_{13}$, as can be achieved in the next round of reactor [23], as an

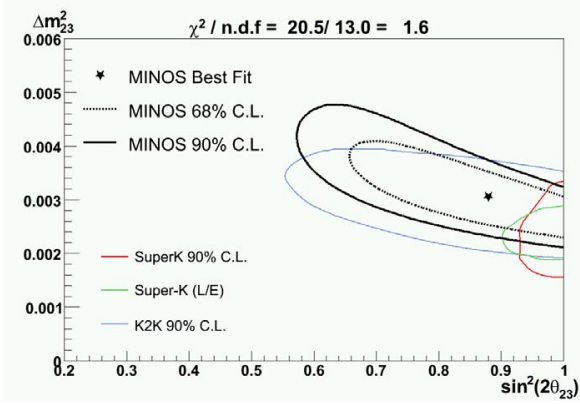

FIG. 4: The allowed regions for $\Delta m_{\mathrm{atm}}^{2}$ and $\sin ^{2}\left(2 \theta_{\mathrm{atm}}\right)$ from atmospheric neutrino data and MINOS accelerator data respectively in red and in black. From Ref. [19].

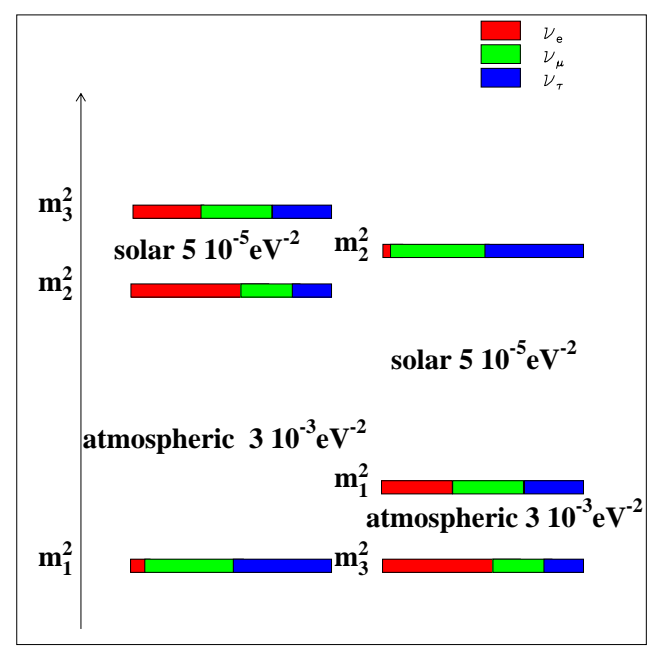

FIG. 5: Ordering of neutrino masses as follows from analysis of neutrino experiments. The colors indicate the proportion of neutrino flavors in each of mass neutrino states, $i=1,2,3$. Notice the strong mixing in the sector 1-2 and 2-3.

example the proposal for Angra [24] and accelerator experiments $[25,26]$.

From all this information we have a picture that neutrinos have different masses, but very tiny compared with other leptons of standard model, but two of three mixing are very strong and one is weaker. All these details can be summarized in Fig. 5. As you can see although you have information about the solar, scale $\Delta m_{\odot}^{2}$ and the mixing angle, $\sin ^{2}\left(\theta_{\odot}\right)$ as well the absolute value atmospheric, scale $\left|\Delta m_{\text {atm }}^{2}\right|$ and the mixing angle $\sin ^{2}\left(2 \theta_{\text {atm }}\right)$, we still don't know about the answer for three questions. What is

1. the value of mixing angle $\theta_{13}$ ?;

2. the hierarchy? The atmospheric scale $\Delta m_{\mathrm{atm}}^{2}$ is greater or smaller then zero?;

3. and did you have $\mathrm{CP}$ violation in the neutrino sector? 


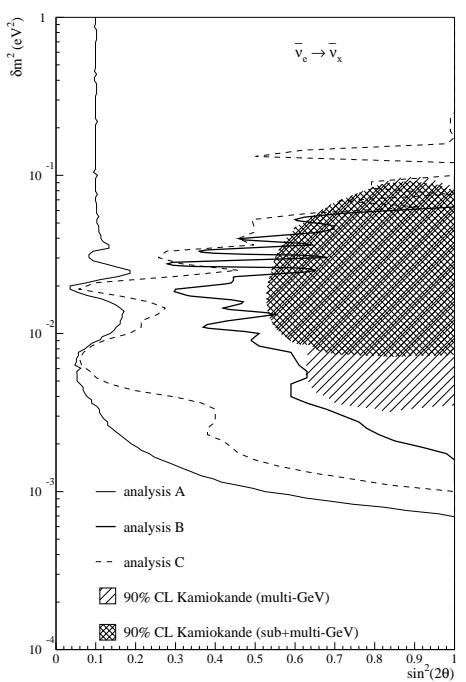

FIG. 6: Exclusion plot contours at $90 \%$ C.L. obtained by the three different analysis by CHOOZ experiment [22].

In another way, the $\mathrm{CP}$ violation angle $\gamma$ is zero or not? The $\mathrm{CP}$ violation phase it is not well constrained by the data [27].

This will our starting point to discuss more complex phenomena and try to elucidate the origin of this lepton number violation phenomena know as neutrino oscillation. Also these if we answer these three question we can but know more clearly what is more suitable to explain these parameters. We have many ideas, but we still we don't know the right track to decide between the plethora of models that we have [28].

\section{NEW DIRECTIONS IN NEUTRINO PHYSICS}

We are beginning to be have sensitivity to more detailed information about the dynamics of neutrino evolution. The basic question, is all dynamics given by Eq. (1) or we can have additional dynamics? All the picture described in previous sections is valid we can have sub-dominant effects that can alter some of neutrino properties.

Examples are new additional states, like sterile states [29] and new interactions, like flavor changing interactions without mass [30], neutrino magnetic momentum without mass [31], flavor changing interactions with mass [32], neutrino magnetic momentum with mass [33] and mass varying neutrinos [34].

One example is the oscillation induced by non-vanishing neutrino magnetic momentum interacting with solar magnetic fields. If neutrinos are Majorana particles these induces neutrino to anti-neutrino conversions. This phenomena is know generally as resonant spin-flip conversion [31, 33, 35-42]. The electromagnetic moment interaction of the Majorana neu-

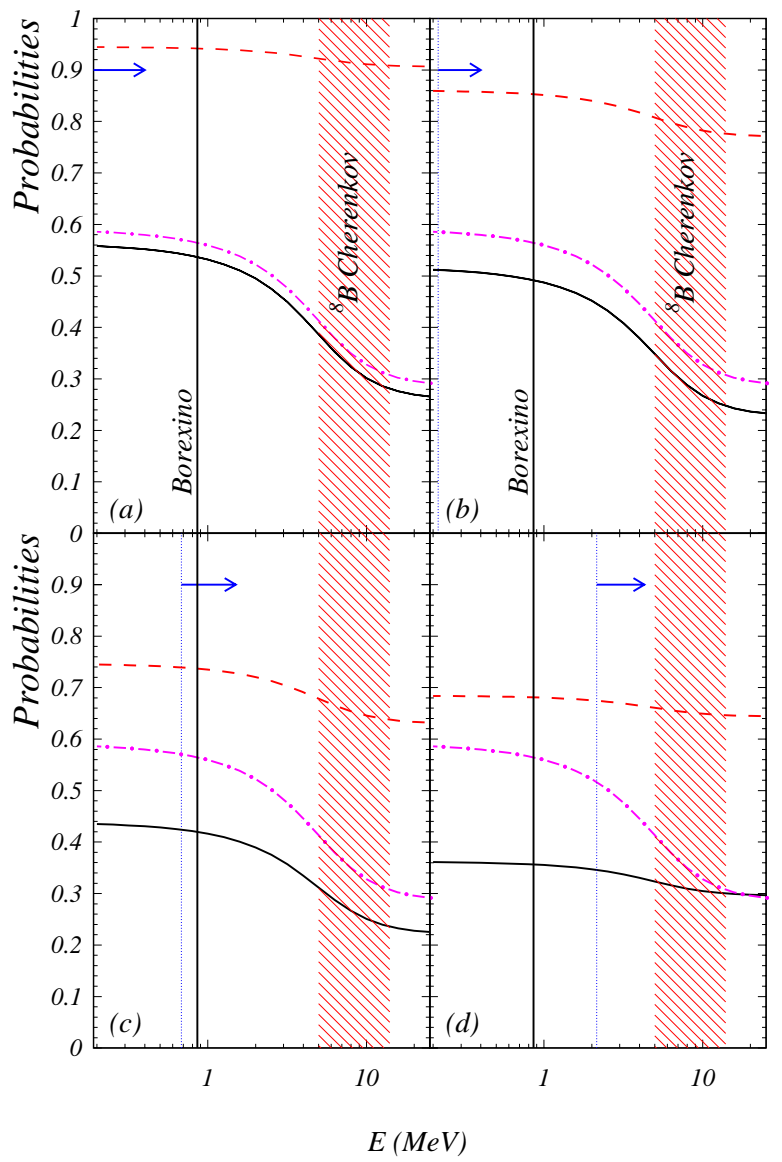

FIG. 7: Neutrino survival probabilities, the solid line is $P\left(v_{e} \rightarrow v_{e}\right)$, the dashed is the sum $P\left(v_{e} \rightarrow v_{e}\right)+P\left(v_{e} \rightarrow v_{\mu}\right)$, and the $P\left(v_{e} \rightarrow \bar{v}_{\tau}\right)$ is the remaining probability until the maximum, as a function of the neutrino energy E. The different panels (a), (b), (c) and (d) respectively correspond to $\mathrm{k}=10^{-15.5} \mathrm{eV}, 10^{-15.0} \mathrm{eV}, 10^{-14.5} \mathrm{eV}$, $10^{-14.0} \mathrm{eV}$. The dot-dashed line is the survival probability for the standard LMA scenario. The vertical solid line denotes the position of Beryllium line. The shallow area denoted the relevant neutrino energy for ${ }^{8} \mathrm{~B}$ neutrino experiments. From Ref. [33].

trinos is given by the following dimension five operator

$$
\mathcal{L}_{E M}=-\frac{1}{2} \mu_{a b} \bar{\Psi}_{a} \Sigma^{\mu v} \Psi_{b} F_{\mu v}
$$

where $\Psi_{a}$ and $\Psi_{b}$ are Majorana spinors of flavors $a$ and $b$ in the 4-component notation, and $\Sigma^{\mu \nu} \equiv i\left[\gamma^{\mu}, \gamma^{v}\right] / 2$, with $\mu$ and $\nu$ being the Lorentz indices. Also $\mu_{a b}$ is the neutrino magnetic momentum between the flavors $a$ and $b$. For Majorana neutrinos, $\mu_{a b} \rightarrow 0$, if $a=b$, then we only have neutrino to antineutrino transitions.

In the standard case, the anti-neutrino evolution matrix is similar to Eq. (1) if we chance $v_{a} \rightarrow \bar{v}_{a}$, where $a$ is any flavor, and $V_{e} \rightarrow-V_{e}$ and $\gamma \rightarrow-\gamma$. In the standard case there is not mixing between neutrinos and anti-neutrinos.

In the case of resonant spin-flavor, we can have neutrino to anti-neutrino conversion. If we choose $\mu_{a b} \neq 0$, for $a$ or $b \equiv e$ to electron neutrino, and zero otherwise we expect to 


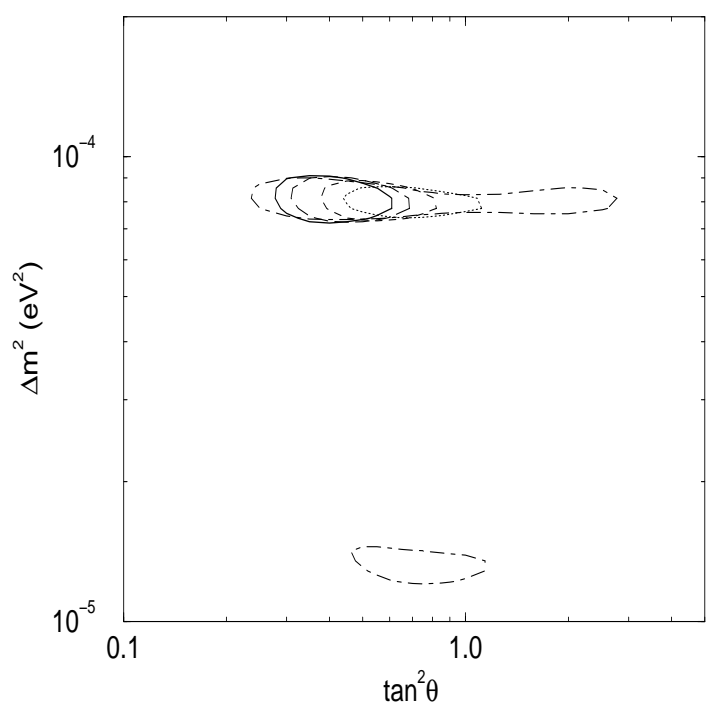

FIG. 8: Combined result for SNO+Super-Kamiokande and KamLAND data, at $99 \%$ C.L.. We use the convention that the black line stands for no magnetic field, the long-dashed line for $k=10^{-15.5} \mathrm{eV}$, the short-dashed line for $k=10^{-15} \mathrm{eV}$, the dotted line for $k=$ $10^{-14.5} \mathrm{eV}$ and the dot-dashed line for $k=10^{-14} \mathrm{eV}$. From Ref. [33].

have $v_{e} \rightarrow \bar{v}_{e}$ conversion. The $\bar{v}_{e}$ flux from the Sun, in turn, is strongly constrained by Super-Kamiokande [43], $\mathrm{SNO}$ [44], and especially KamLAND [45] which can detect $v_{e} \rightarrow \bar{v}_{e}$ conversion of the solar ${ }^{8} \mathrm{~B}$ neutrinos at the level of a few hundredths of a percent. This ruled out any sizable influence of spin-flavor mechanism in the neutrino dynamics.

If we can choose $\mu_{a b} \neq 0$, for $a(b) \equiv \mu(\tau)$, in this case these implies that $v_{e} \rightarrow \bar{v}_{\mu}\left(\bar{v}_{\tau}\right)$ conversion. In this case, the above limit did not apply. If so, we expect to see a contribution of $\bar{v}_{\mu}\left(\bar{v}_{\tau}\right)$ neutrinos coming from the sun, depending of the size of magnetic field. For regular magnetic fields, the effect is too small it is very similar to standard case without $\bar{v}_{\mu}\left(\bar{v}_{\tau}\right)$ neutrinos.

If we assume the presence of random magnetic fields, present in the outer layers of the Sun with average values equal to zero $\langle B(t)\rangle \rightarrow 0$, but squared average values non zero, $\left\langle B(t) B\left(t^{\prime}\right)\right\rangle \neq 0$. Let's define the parameter to quantify the magnitude of random magnetic fields,

$$
k \equiv 1.7 \times 10^{-17}\left[\frac{\mu_{\mu \tau}}{10^{-11} \mu_{B}}\right]^{2}\left[\frac{B^{\text {rand }}}{1 \mathrm{MG}}\right]^{2}\left[\frac{D_{0}}{1 \mathrm{~km}}\right] \mathrm{eV},
$$

and $D_{0}$ is the coherence length of the random magnetic field. If $k \rightarrow 0$, then you the standard case, otherwise you will a production of anti muon(tauon) neutrinos from the sun. You can see in Fig. 7 the probabilities.

Now we can test this scenario in the solar+KamLand experiments, the final result is shown in Fig. (8). As a consequence, a totally new region of compatibility between solar neutrinos and KamLAND, which we call very-low LMA, appears at $99 \%$ C.L. for small values of $\Delta m_{\odot}^{2} \sim[1-2] \times 10^{-5} \mathrm{eV}^{2}$ and maximal mixing.

\section{CONCLUSIONS}

We have made a review of the status of neutrino physics, in particular of new phenomena know as neutrino oscillation. We have shown that the neutrino oscillation was shown in many different experiments that can be made compatible if we have three standard neutrinos that have different masses and that mix between each other.

We have show a consistent picture of two different scales, the so called solar scale, $\Delta m_{\odot}^{2} \sim 8 \times 10^{-5} \mathrm{eV}^{2}$ and the atmospheric scale the atmospheric scale, $\Delta m_{\mathrm{atm}}^{2} \sim 2.5 \times$ $10^{-3} \mathrm{eV}^{2}$ as showed in Fig. 5. The respective mixing angles, the solar $\theta_{\odot}$ and the atmospheric $\theta_{\text {atm }}$ are larger. The only unknown angle $\theta_{31}$ is small compared with the others. We still have many unknowns things that we will like to discover,

1. the value of mixing angle $\theta_{13}$ ?;

2. the hierarchy? The atmospheric scale $\Delta m_{\mathrm{atm}}^{2}$ is greater or smaller then zero?;

3. and did you have CP violation in the neutrino sector? In another way, the $\mathrm{CP}$ violation angle $\gamma$ is zero or not?

Beside that, you can use neutrino to test new states or new interactions as in the example of the resonant spin-flip conversion with neutrino magnetic momentum between the muon and tauon flavors, $\mu_{\mu \tau}$. In this case we show that we can have a sizable $v_{e} \rightarrow \bar{v}_{\mu}\left(\bar{v}_{\tau}\right)$ conversion and we can modify the allowed region for the solar parameters, $\Delta m_{\odot}^{2}$ and $\theta_{\odot}$.

We hope that in few years you can answer all questions commented here, and shed some light about the nature of neutrino conversion as can be possible in the next generation of neutrino experiments, such as MINOS experiment $[19,46]$ for pinpoint the mass scale $\Delta m_{\mathrm{atm}}^{2}$ parameter, the SADO proposal [47] for improving the mixing angle $\theta_{\odot}$, DOUBLECHOOZ experiment [48] and Angra Neutrino proposal [49] to measure the mixing angle $\theta_{13}$.

\section{Acknowledgments}

This work was partially supported by Fundação de Amparo à Pesquisa do Estado de São Paulo (FAPESP) and Conselho Nacional de Desenvolvimento Científico e Tecnológico (CNPq). I thank the organizers of the XXVI Encontro Nacional de Física de Partículas e Campos for the very warm and friendly environment.
[1] S. L. Glashow, Nucl. Phys. 22, 579 (1961); S. Weinberg, Phys. Rev. Lett. 19, 1264 (1967); A. Salam, "Elementary Particle
Theory: Relativistic Groups and Analitycity", Nobel Sympo- 
sium No.8, Ed. N. Svartholm (Almqvist and Wiksells, Stockholm, 1968); S. Weinberg, Rev. Mod. Phys. 52, 515 (1980); A. Salam, Rev. Mod. Phys. 52, 525 (1980); S. L. Glashow, Rev. Mod. Phys. 52, 539 (1980).

[2] B. Pontecorvo, Sov. Phys. JETP 6, 429 (1957) [Zh. Eksp. Teor. Fiz. 33, 549 (1957)]; B. Pontecorvo, Sov. Phys. JETP 26, 984 (1968)[Zh. Eksp. Teor. Fiz. 531717 (1967)]; V. N. Gribov and B. Pontecorvo, Phys. Lett. B 28, 493 (1969).

[3] Z. Maki, M. Nakagawa and S. Sakata, Prog. Theor. Phys. 28, 870 (1962);

[4] C. L. Cowan, Jr., F. Reines, F. B. Harrison, H. W. Kruse, and A. D. McGuire, Science 124, 103 (1956).

[5] Kamiokande Collaboration, H. S. Hirata et al., Phys. Lett. B 205, 416 (1988); ibid. 280, 146 (1992); Y. Fukuda et al., ibid. 335, 237 (1994).

[6] IMB Collaboration, R. Becker-Szendy et al., Phys. Rev. D 46, 3720 (1992).

[7] Soudan-2 Collaboration, W. W. M. Allison et al., Phys. Lett. B 391, 491 (1997)

[8] Super-Kamiokande Collaboration, Y. Fukuda et al., Phys. Rev. Lett. 81, 1562 (1998); Phys. Lett. B 436, 33 (1999).

[9] Amanda Collaboration, E. Andrés et al., Nature 410, 441 (2000).

[10] Homestake Collaboration, B. T. Cleveland et al., Astroph. J. 496, 505 (1998).

[11] Kamiokande Collaboration, Y. Fukuda et al., Phys. Rev. Lett. 77, 1683 (1996)

[12] GALLEX Collaboration, W. Hampel et al., Phys. Lett. B 447, 127 (1999).

[13] GNO Collaboration, M. Altmann et al., Phys.Lett. B 616, 174 (2005).

[14] SAGE Collaboration, V. N. Gavrin in behalf of SAGE Collaboration, Nucl. Phys. Proc. Suppl. 138, 87 (2005).

[15] Super-Kamiokande Collaboration, S. Fukuda et al., Phys. Rev. Lett. 86, 5651 (2001); Phys. Lett. B 539, 179 (2002); M. B. Smy et al., Phys. Rev. D 69, 011104 (2004).

[16] SNO Collaboration, Q. R. Ahmad et al., Phys. Rev. Lett. 87, 071301 (2001); 89, 011301 (2002); 89, 011302 (2002); S. N. Ahmed et al., 92, 181301 (2004).

[17] KamLAND Collaboration, K. Eguchi et al., Phys. Rev. Lett. 90, 021802 (2003); T. Araki et al., 94, 081801 (2005).

[18] K2K Collaboration, M. H. Ahn et al., Phys. Rev. Lett. 90, 041801 (2003)

[19] D. Petyt talk at Joint Experimetal-Theoretical Seminar at FERMILAB ; An online version is avaliable at http://www-numi.fnal.gov/talks/results06.html .

[20] S. Eidelman et al., Phys. Lett. B592, 1 (2004).

[21] L. Wolfenstein, Phys. Rev. D 17, 2369 (1978); S. P. Mikheyev and A. Yu. Smirnov, Nuovo Cim. C 9, 17 (1986).

[22] CHOOZ Collaboration, M. Apollonio et al., Phys. Lett. B 420, 397 (1998); ibid. B 466, 415 (1999); Eur. Phys. J. C27, 331 (2003).

[23] K. Anderson et al., arXiv:hep-ex/0402041; See also talks at Fourth Workshop on Future Low Energy Experiments, Hotel do Frade, Angra dos Reis (RJ), Brazil, Feb. 23-25 2005. Talks available at http://www.ifi.unicamp.br/ lenews05/.

[24] J. C. Anjos, A. F. Barbosa, R. Zukanovich Funchal, E. Kemp, J. Magnin, H. Nunokawa, O. L. G. Peres, D. Reyna, and R. C. Shellard, Nucl. Phys. Proc. Suppl. 155, 231 (2006).

[25] T2K Collaboration, Y. Itow et al., arXiv:hep-ex/0106019, Homepage: http://neutrino.kek.jp/jhfnu/.

[26] NOvA Collaboration, D. Ayres et al., arXiv:hep-ex/0503053; Homepage at http://www-nova.fnal.gov/.

[27] O. L. G. Peres and A. Yu. Smirnov, Nucl. Phys. B 680, 479 (2004).

[28] R. N. Mohapatra et al., "Theory of neutrinos: A white paper," arXiv:hep-ph/0510213.

[29] P. C. de Holanda and A. Yu. Smirnov, Phys. Rev. D 69, 113002 (2004).

[30] M. M. Guzzo, H. Nunokawa, P. C. de Holanda, and O. L. G. Peres, Phys. Rev. D 64, 097301 (2001).

[31] A. M. Gago, M. M. Guzzo, P. C. de Holanda, H. Nunokawa, O. L. G. Peres, V. Pleitez, and R. Zukanovich Funchal, Phys. Rev. D 65, 073012 (2002).

[32] M. M. Guzzo, P. C. de Holanda, and O. L. G. Peres, Phys. Lett. B 591, 1 (2004).

[33] M. M. Guzzo, P. C. de Holanda, and O. L. G. Peres, Phys. Rev. D 72, 073004 (2005).

[34] M. C. Gonzalez-Garcia, P. C. de Holanda, and R. Zukanovich Funchal, Phys. Rev. D 73, 033008 (2006).

[35] C. S. Lim and W. J. Marciano, Phys. Rev. D 37, 1368 (1988).

[36] E. K. Akhmedov, Phys. Lett. B 213, 64 (1988).

[37] A. B. Balantekin, P. J. Hatchell and F. Loreti, Phys. Rev. D 41, 3583 (1990).

[38] M. M. Guzzo and H. Nunokawa, Astropart. Phys. 12, 87 (1999).

[39] E. K. Akhmedov and J. Pulido, Phys. Lett. B 553, 7 (2003).

[40] A. Friedland and A. Gruzinov, Astropart. Phys. 19, 575 (2003).

[41] J. Barranco, O. G. Miranda, T. I. Rashba, V. B. Semikoz, and J. W. F. Valle, Phys. Rev. D 66, 093009 (2002).

[42] B. C. Chauhan and J. Pulido, JHEP 0412, 040 (2004).

[43] Super-Kamiokande Collaboration, Y. Gando et al., Phys. Rev. Lett. 90, 171302 (2003).

[44] SNO Collaboration, B. Aharmim et al., Phys. Rev. D 70, 093014 (2004)

[45] KamLAND Collaboration, K. Eguchi et al., Phys. Rev. Lett. 92, 071301 (2004)

[46] Philipe Gouffon in behalf of MINOS Collaboration, these proceedings.

[47] H. Minakata, H. Nunokawa, W. J. C. Teves, and R. Zukanovich Funchal, Phys. Rev. D 71, 013005 (2005); H. Minakata, H. Nunokawa, W. J. C. Teves, and R. Zukanovich Funchal, Nucl. Phys. Proc. Suppl. 145, 45 (2005).

[48] F. Ardellier et al., arXiv:hep-ex/0405032; S. Berridge et al., arXiv:hep-ex/0410081.

[49] J. C. Anjos, in behalf of Angra Neutrino Collaboration, these proceedings. 